\title{
On the interactions between molecules in an off-resonant laser beam: Evaluating the response to energy migration and optically induced pair forces
}

\author{
David L. Andrews ${ }^{a)}$ and Jamie M. Leeder \\ Nanostructures and Photomolecular Systems, School of Chemical Sciences, University of East Anglia, \\ Norwich NR4 7TJ, United Kingdom
}

(Received 24 October 2008; accepted 11 December 2008; published online 20 January 2009)

\begin{abstract}
Electronically excited molecules interact with their neighbors differently from their ground-state counterparts. Any migration of the excitation between molecules can modify intermolecular forces, reflecting changes to a local potential energy landscape. It emerges that throughput off-resonant radiation can also produce significant additional effects. The context for the present analysis of the mechanisms is a range of chemical and physical processes that fundamentally depend on intermolecular interactions resulting from second and fourth-order electric-dipole couplings. The most familiar are static dipole-dipole interactions, resonance energy transfer (both second-order interactions), and dispersion forces (fourth order). For neighboring molecules subjected to off-resonant light, additional forms of intermolecular interaction arise in the fourth order, including radiation-induced energy transfer and optical binding. Here, in a quantum electrodynamical formulation, these phenomena are cast in a unified description that establishes their inter-relationship and connectivity at a fundamental level. Theory is then developed for systems in which the interplay of these forms of interaction can be readily identified and analyzed in terms of dynamical behavior. The results are potentially significant in Förster measurements of conformational change and in the operation of microelectromechanical and nanoelectromechanical devices. (C) 2009 American Institute of Physics. [DOI: 10.1063/1.3062872]
\end{abstract}

\section{INTRODUCTION}

\section{A. Energy migration and its effects}

The primary result of photon absorption in any complex dielectric material is the population of electronic excited states in individual atomic or molecular sites. Typically, each such absorption is followed by a rapid but partial degradation of the acquired energy, dissipative losses due to intramolecular, or lattice nuclear vibrations ultimately being manifested in the form of heat. The majority of the excitation energy, held in a localized electronic excited state, may be acquired by a neighboring atom or molecule with a suitably disposed electronic state through a process known as resonance energy transfer (RET). ${ }^{1}$

RET operates across a chemically diverse and extensive range of material systems. Its most widely known role is in the channeling of electronic excitation in photosynthetic systems $^{2-4}$ and their biomimetic analogs. ${ }^{4-7}$ The phenomenon also has an important function in the operation of organic light-emitting diodes and luminescence detectors. ${ }^{8,9}$ In the realm of molecular biology, the determination of protein structures and the characterization of dynamical processes are furthered by studies of the transfer of energy between chromophores; ${ }^{10-12}$ several ultrasensitive molecular imaging applications are again based on the same underlying principle. $^{13-17}$

The migration of electronic excitation between molecu-

${ }^{a)}$ Electronic mail: d.1.andrews@uea.ac.uk. lar units, central to each such application, has received extensive experimental and theoretical study. Its spectroscopic manifestations, in particular, are well characterized. Much less attention has been paid to the nanoscale mechanical effects accompanying RET. As recent preliminary studies have shown, participating donor and acceptor species must generally experience a displacement from their normal equilibrium position accompanying and caused by the translation of energy. ${ }^{18,19}$ Moreover, the typical magnitude of such effects falls well within the current limits of experimental detection using atomic force microscopy and related equipment. A full theoretical treatment of this phenomenon is the subject of the present study; the analysis is extended to accommodate and appraise subsidiary effects due to throughput radiationspecifically, additional mechanical and dynamical effects that arise on the propagation of off-resonant light through the transfer system.

\section{B. The theoretical framework}

To address the theory of energy transfer with rigor we employ the methods of molecular quantum electrodynamics (QED). ${ }^{20-22}$ Analysis by QED signifies the application of quantum mechanical principles to both matter and electromagnetic field, correctly accommodating transitions in both molecular and radiation states. Notable accomplishments of the theory include (but are not limited to) the successful theoretical determination of the Lamb shift for both light and superheavy elements, ${ }^{23-25}$ the magnitude of the magnetic moment of the electron, ${ }^{24,26}$ and an accurate determination of 
the fine structure constant. ${ }^{24,26}$ The theory has been tested to a higher degree of precision than any other in modern physics, still being verified by the most accurate experimental measurements. ${ }^{24,26}$

One of the hallmarks of QED-in contrast to both classical and semiclassical representations-is that it furnishes each mode of a fully quantized radiation field with a zeropoint energy, consistent with quantum fluctuations in the corresponding electric and magnetic fields. As a physical consequence, these vacuum fields give rise to electromagnetic field quanta that can contribute to the dynamical behavior of a system. The dispersion interaction between electrically neutral molecules affords a good example. The short-range $R^{-6}$ potential can be delivered by calculations performed on either a semiclassical or QED basis, assuming the absence of observable light. However, in the QED calculations, based on coupling by the vacuum field quanta, the form of the interaction potential exhibits retardation effects-precisely because the field quanta propagate at the finite speed of light. The result is a change to an $R^{-7}$ dependence at large distances - and the success of this interpretation is vindicated by experimental measurements that have verified this prediction. ${ }^{27}$

The application of QED greatly facilitates the identification of fundamental links between effects that are physically different but share a common form of mathematical development. An obvious example is the similarity in the theoretical constructs of Raman scattering and two-photon absorption ${ }^{21}$ whose mathematical isomorphism is mirrored in less familiar, static field-induced, and cooperative effects. ${ }^{28,29}$ Another case is the formal link between fluorescence resonance energy transfer (FRET) and sequential Raman scattering. ${ }^{30}$

While some of the above examples are of primarily theoretical interest, the broader purpose of the present analysis is to address a system in which various physical effects are not only mathematically but also physically interlinked. Calculations accommodating both mechanical forces and electronic processes, accurate to fourth order in perturbation theory, have not to our knowledge been attempted before. The results prove to exhibit a variety of effects that are amenable to experimental measurement.

\section{QED FOUNDATION}

For a QED analysis, discussion generally begins with the complete system Hamiltonian

$$
H=\sum_{\xi} H_{\mathrm{mol}}^{(\xi)}+\sum_{\xi} H_{\mathrm{int}}^{(\xi)}+H_{\mathrm{rad}} .
$$

Here, $H_{\text {rad }}$ is the fully quantized radiation Hamiltonian and $H_{\mathrm{mol}}^{(\xi)}$ corresponds to the molecular Hamiltonian for a molecule labeled $\xi$; this operator includes terms relating to both nuclei and electrons. Matter and radiation states couple through the interaction Hamiltonian $H_{\text {int }}^{(\xi)}{ }^{21,31}$ Explicitly, the electric interactions effected by $H_{\text {int }}^{(\xi)}$ can be represented in the following generalized multipolar form, cast using the convention of summation over repeated Cartesian (subscript) indices: $:^{32,33}$

$$
H_{\text {int }}^{(l)}(\xi)=-\varepsilon_{0}^{-1} E_{i_{1} i_{2} \cdots i_{l}}^{(l)}(\xi) \nabla_{i_{2}} \cdots \nabla_{i_{l}} d_{i_{1}}^{\perp}\left(\boldsymbol{R}_{\xi}\right),
$$

with $E_{i_{1} i_{2} \cdots i_{l}}^{(l)}(\xi)$ representing the $l$ th order electric multipole operator, operating on system molecular states. A characteristic of the multipolar formalism is the absence of any direct molecule-molecule interaction. The inclusion of the quantized field determines that intermolecular interactions are mediated solely by the exchange of photons. ${ }^{34-36}$ The transverse electric field operator $\boldsymbol{d}^{\perp}\left(\boldsymbol{R}_{\xi}\right)$ is at position vector $\boldsymbol{R}_{\xi}$ acting on the radiation states:

$$
\begin{aligned}
\boldsymbol{d}^{\perp}\left(\boldsymbol{R}_{\xi}\right)= & \sum_{\boldsymbol{p}, \lambda}\left(\frac{\hbar c p \varepsilon_{0}}{2 V}\right)^{1 / 2} i\left[\boldsymbol{e}^{(\lambda)}(\boldsymbol{p}) a^{(\lambda)}(\boldsymbol{p}) \exp \left(i \boldsymbol{p} \cdot \boldsymbol{R}_{\xi}\right)\right. \\
& \left.-\overline{\boldsymbol{e}}^{(\lambda)}(\boldsymbol{p}) a^{\dagger(\lambda)}(\boldsymbol{p}) \exp \left(-i \boldsymbol{p} \cdot \boldsymbol{R}_{\xi}\right)\right] .
\end{aligned}
$$

Above, $\boldsymbol{e}^{(l)}(\boldsymbol{p})$ is the electric field unit vector with complex conjugate $\overline{\boldsymbol{e}}^{(l)}(\boldsymbol{p})$ and $V$ is an arbitrary quantization volume. The presence of the photon annihilation and creation operators $a$ and $a^{\dagger}$ determines that each operation of $H_{\text {int }}^{(\xi)}$ destroys or creates a single photon-noting that virtual photons are to be summed over all wave vectors $\boldsymbol{p}$ and polarizations $\lambda$.

Providing that matter and field coupling is sufficiently small with respect to intramolecular bond energies, i.e., the propagating radiation does not disrupt the molecular structure of any participant within the system, the physical consequence of $H_{\text {int }}^{(\xi)}$ can be treated as a perturbation, partitioned from the unperturbed sum of all molecular and radiation operators, $H_{0}$ :

$$
H=H_{0}+\sum_{\xi} H_{\mathrm{int}}^{(l)}(\xi) .
$$

Assuming that both the quantized molecular and radiation terms in Eq. (1) are known, $H_{\text {mol }}^{(\xi)}$ being the sum of potential and kinetic energy operators and $H_{\text {rad }}$ determined by the quantized electric displacement and magnetic field, ${ }^{21,31}$ the eigenstates $|Q\rangle$ of $H_{0}$ are defined by

$$
|Q\rangle=\left|\operatorname{mol}_{Q} ; \operatorname{rad}_{Q}\right\rangle .
$$

These states represent the basis within which the effect of perturbative coupling is to be determined. The perturbation is cast as an infinite series in terms of a quantum probability amplitude or matrix element $M_{F I}$ connecting initial and final system states $|I\rangle$ and $|F\rangle$, respectively: $:^{37}$

$$
M_{F I}=\sum_{q=1}^{\infty}\left\langle F\left|H_{\mathrm{int}}^{(\xi)}\left(T_{0} H_{\mathrm{int}}^{(\xi)}\right)^{q-1}\right| I\right\rangle .
$$

Above, $T_{0} \approx\left(E_{I}-H_{0}\right)^{-1}$, with $E_{I}$ the initial system energy. The parameter $q$, which denotes the power of $H_{\text {int }}^{(\xi)}$ in each term of the expansion, has significant physical meaning; as a result of the photon creation and annihilation operators defined in Eq. (3) it corresponds to the number of fundamental (real or virtual) photon-matter interactions. Where $M_{F I}$ describes a physically observable process, application of Fermi's golden rule allows the transition rate between the initial and final states of the system to be determined: $:^{33,36}$ 


$$
\Gamma=\frac{2 \pi \rho}{\hbar}\left|M_{F I}\right|^{2},
$$

where $\rho$ is the density of molecular states in the electronic level of the acceptor populated by the energy transfer.

In the following, the theory of second- and fourth-order interactions is developed with a particular focus on elucidating the effects of intermolecular energy transfer. All interactions are considered to occur beyond the region of significant wave function overlap. The entire system is considered to be isolated; its Hamiltonian is duly Hermitian and time independent, and the system eigenstates have time-independent energies. As a consequence, dynamical processes within the system are uniquely associated with a response to the intermolecular migration of energy and to any time-varying radiative input—such as the pulsed off-resonant laser radiation that proves to induce features of particular interest.

\section{COUPLING PROCESSES}

To ensure rigorous inclusion of all processes and mechanisms to a common and consistent level, we evaluate up to the fourth order of perturbative expansion all relevant couplings between physically identifiable system states. One overriding condition is that the final state of every radiation mode is identical to its initial state, i.e., no net absorption or emission of radiation occurs. For this reason, since $H_{\text {int }}^{(\xi)}$ from Eqs. (2) and (3) can only create or destroy one photon on each operation, only even values of the power index $q$ arise. Hence the leading nonzero terms in the series expressed by Eq. (6) can be developed through insertion of the state completeness relation on the right-hand side of each $T_{0}$ operator:

$$
\begin{aligned}
M_{F I}= & \sum_{R} \frac{\left\langle F\left|H_{\mathrm{int}}^{(\xi)}\right| R\right\rangle\left\langle R\left|H_{\mathrm{int}}^{(\xi)}\right| I\right\rangle}{\left(E_{I}-E_{R}\right)} \\
& +\sum_{R, S, T} \frac{\left\langle F\left|H_{\mathrm{int}}^{(\xi)}\right| T\right\rangle\left\langle T\left|H_{\mathrm{int}}^{(\xi)}\right| S\right\rangle\left\langle S\left|H_{\mathrm{int}}^{(\xi)}\right| R\right\rangle\left\langle R\left|H_{\mathrm{int}}^{(\xi)}\right| I\right\rangle}{\left(E_{I}-E_{R}\right)\left(E_{I}-E_{S}\right)\left(E_{I}-E_{T}\right)}+\cdots,
\end{aligned}
$$

with $|R\rangle,|S\rangle$, and $|T\rangle$ representative of intermediate states, each denominator term being the energy difference between one of these intermediates and the initial state.

\section{A. Second-order processes}

\section{Static dipole interaction}

From Eq. (8), the leading contribution $(q=2)$ represents a second-order perturbation, which in the short-range signifies the creation and annihilation of a single virtual photon. The simplest case is the static interaction of two ground-state molecules with permanent electric-dipole moments, as represented in Fig. 1 by the usual time-ordered (Feynman) diagrams. Deploying the dipole term in Eq. (2), these diagrams elucidate two possible contributions to the interaction, the first entailing the creation of a virtual photon at molecule $A$ and subsequent annihilation at $B$ [Fig. 1(a) $]$ and the second case the reverse [Fig. 1(b)]. As with all subsequently described processes, a complete description requires a summation of the quantum amplitudes delivered by all such topo-

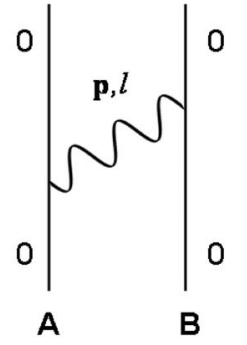

(a)

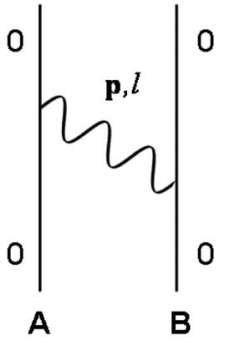

(b)
FIG. 1. Both possible time-ordered Feynman representations of the static coupling between molecules $A$ and $B$ through the propagation of a single virtual photon, time progressing upwards.

logically distinct representations. The result emerges as follows, where $\mu^{00}$ signifies a static electric-dipole moment:

$$
\Delta E=\mu_{i}^{00(A)} \mu_{j}^{00(B)} V_{i j}(0),
$$

invoking the short-range coupling tensor $V_{i j}(0)$ :

$$
V_{i j}(0)=\frac{1}{4 \pi \varepsilon_{0} R^{3}}\left(\delta_{i j}-3 \hat{R}_{i} \hat{R}_{j}\right) .
$$

Equation (10) is of course the standard dipole-dipole coupling formula. Its inclusion here, both for completeness and by way of introduction to the more complex forms of coupling detailed below, also identifies the involvement of the static (or short-range) limit of a general retarded dipoledipole coupling tensor $V$ that plays a central role in those other processes. Note that in the investigation of dynamical behavior in Sec. IV, the assumption of a system comprising nonpolar molecules means that no role is played by static dipole coupling itself.

\section{Resonance energy transfer}

In terms of an experimentally observable process, the simplest intermolecular interaction is the transfer of energy through resonance coupling between molecules, one of which is in an initially prepared excited state. As with static dipole coupling, RET is a second-order interaction exhibiting two photon-matter interactions, i.e., it involves the propagation of a single virtual photon, see Fig. 2. The matrix element for RET is the first contribution in Eq. (8). Using the labels $0, \alpha$, and $\beta$ to represent the electronic ground and corresponding excited states of the donor and acceptor, respec-

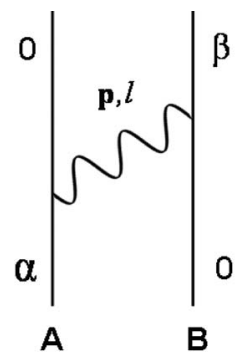

(a)

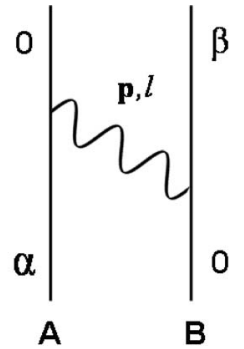

(b)
FIG. 2. Feynman diagrams illustrating RET by virtual photon transfer. Diagram (a) illustrates virtual photon creation at $A$ and subsequent annihilation at $B$ with diagram (b) representing the opposite evolution. 
tively, the initial, final, and intermediate states for RET are defined thus:

$$
\begin{aligned}
& |I\rangle=\left|A_{\alpha}, B_{0} ; 0\right\rangle, \quad\left|R_{1}\right\rangle=\left|A_{0}, B_{0} ; 1(\boldsymbol{p}, \lambda)\right\rangle, \\
& \left|R_{2}\right\rangle=\left|A_{\alpha}, B_{\beta} ; 1(\boldsymbol{p}, \lambda)\right\rangle, \quad|F\rangle=\left|A_{0}, B_{\beta} ; 0\right\rangle .
\end{aligned}
$$

In Eq. (11), the summed intermediate states $|R\rangle$ of Eq. (8) have been explicitly cast in each of two permissible forms, one virtual photon being present in each. Respectively, $\left|R_{1}\right\rangle$ and $\left|R_{2}\right\rangle$ relate to conditions where either both molecules are or neither is in the electronic ground state. The following second-order RET matrix element is determined from the first term of Eq. (8), its full derivation being already well documented, ${ }^{32,33,36}$ leading to the following result:

$$
M_{F I}^{(2)}=\mu_{i}^{0 \alpha(A)} \mu_{j}^{\beta 0(B)} V_{i j}(p, \boldsymbol{R}),
$$

where transition dipole components are denoted in the form $\mu_{i}^{\eta r} \equiv\left\langle\eta\left|\mu_{i}\right| r\right\rangle$. The fully retarded coupling tensor of rank 2, $V_{i j}(p, \boldsymbol{R})$, is exactly expressible as

$$
\begin{aligned}
V_{i j}^{ \pm}(p, \boldsymbol{R})= & \frac{\exp (\mp i p R)}{4 \pi \varepsilon_{0} R^{3}} \\
& \times\left[\left(\delta_{i j}-3 \hat{R}_{i} \hat{R}_{j}\right)(1 \pm i p R)-\left(\delta_{i j}-\hat{R}_{i} \hat{R}_{j}\right)(p R)^{2}\right],
\end{aligned}
$$

noting that both positive and negative imaginary contributions are acceptable (producing identical results for the observable rate); the latter form is more commonly cited. ${ }^{38}$ For significantly small distances, i.e., where $p R \ll 1$, the coupling tensor in Eq. (13) essentially reduces to a short-range limit equivalent to the coupling of static dipoles [Eq. (10)] dependent on $R^{-3}$. Conventionally RET is thus described as being "radiationless" in the short range, a process induced by instantaneous Coulombic interaction. One advantage of the QED formulation is that it establishes a seamless linkage with the longer-range, ultimately radiative behavior. ${ }^{38,39}$

\section{B. Fourth-order processes}

\section{Casimir-Polder (dispersion) interaction}

Considered next are interactions governed by a total of four photon-matter events. In the absence of an applied electromagnetic field, the simplest and most widely relevant example is the Casimir-Polder dispersion interaction. In QED terms, this interaction is mediated by the intermolecular propagation of two virtual photons, ${ }^{21,34,40}$ associated with the second term in Eq. (8). The coupling is illustrated by 12 distinct Feynman diagrams (see Fig. 3) which identify the possible intermediate states $r$ and $s$ for $A$ and $B$, respectively. As an example, for the attractive coupling between groundstate molecules, the following molecular and radiation states define the contribution from Fig. 3(c):

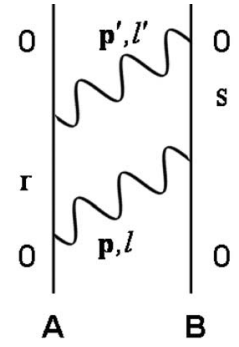

(a)

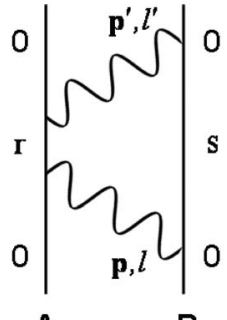

(b)

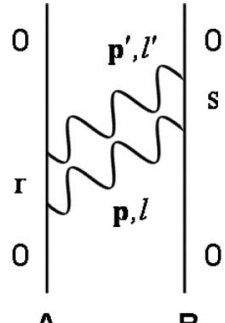

(c)
FIG. 3. Three of 12 distinct time-ordered representations of the fourth-order dispersion force. Process involves migration of two virtual photons from $A$ and $B$ or one from each.

$$
\begin{aligned}
& |I\rangle=|F\rangle=\left|A_{0}, B_{0} ; 0\right\rangle, \quad|R\rangle=\left|A_{r}, B_{0} ; 1(\boldsymbol{p}, \lambda)\right\rangle, \\
& |S\rangle=\left|A_{0}, B_{0} ; 1(\boldsymbol{p}, \lambda), 1\left(\boldsymbol{p}^{\prime}, \lambda^{\prime}\right)\right\rangle, \\
& |T\rangle=\left|A_{0}, B_{s} ; 1\left(\boldsymbol{p}^{\prime}, \lambda^{\prime}\right)\right\rangle .
\end{aligned}
$$

For brevity, rather than consider all possible time orderings required to define the complete potential for any distance, we instead consider two limits that exhibit a strikingly different response.

In the near-zone (where intermolecular distances are small compared to the longest wavelengths of absorption or fluorescence) the coupling is essentially instantaneous. The uncertainty principle dictates that the short-lived virtual photons may accordingly exhibit energies that are large compared to the molecular transition energies. This acts as a constraint upon the time-ordered contributions that contribute significantly to the dispersion interaction. ${ }^{21,41}$ The sum of all such contributions determines a limiting result that is more conventionally obtained using a dipolar coupling approximation (Appendix) with second-order perturbation theory. For freely rotating molecules, the dispersion interaction yields a result dependent on $R^{-6}$, commonly referred to as the London potential when both donor and acceptor are in the electronic ground state:

$$
\Delta E=-\frac{1}{24 \pi^{2} \varepsilon_{0}^{2} R^{6}} \sum_{r, s} \frac{\left|\boldsymbol{\mu}^{0 r(A)}\right|^{2}\left|\boldsymbol{\mu}^{0 s(B)}\right|^{2}}{\left(E_{r 0}^{A}+E_{s 0}^{B}\right)} .
$$

Conversely, in the far-zone limit, increasing propagation time allows the virtual photons to convey lower energies, and the calculations are dominated by contributions consistent with photon frequencies that are small with respect to the molecular absorption and emission frequencies. It is in this distance range that retardation effects become apparent, the duly modified dispersion interaction now varying as $R^{-7}$. $^{40-42}$ For calculational simplicity, all interactions are subsequently discussed within the near-field range; the effects to be described are certainly most prominent in this region.

\section{Optically induced pair forces}

In the presence of intense off-resonant laser light, additional intermolecular effects are manifested as a result of real photon-matter interactions. For the identification of such effects, calculations are performed on a basis state for which the occupation number of at least one photon mode is 


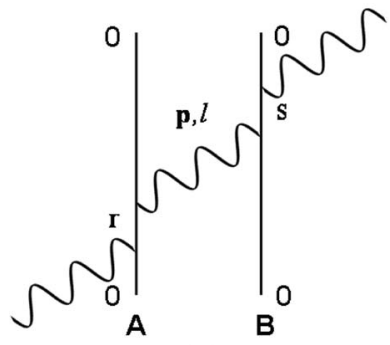

(a)

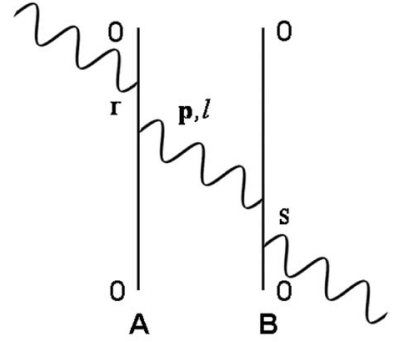

(b)
FIG. 4. Two of the 48 distinct illustrations of the fourth-order optically induced pair force. Represented above are (a) real photon absorption at $A$ coupled by migration of a virtual photon annihilated at $B$, with stimulated re-emission of a real photon back into the auxiliary field. The opposite process is represented by (b).

nonzero. ${ }^{43,44}$ In order to determine energy shifts arising from a coupling with throughput radiation, it is necessary to identify terms that are diagonal in this basis, taking the following form:

$$
|I\rangle=|F\rangle=\left|A_{0}, B_{0} ; n(\boldsymbol{p}, l)\right\rangle .
$$

The leading contribution to the interaction modified by laser input is a fourth-order perturbation, described as real photon annihilation at the donor and stimulated re-emission from the acceptor (or vice versa), with both molecules coupled by a single virtual photon-see Fig. 4. In accordance with energy conservation the throughput radiation suffers no overall change.

The analysis of an optically induced pair energy shift begins from the second contribution of Eq. (8) - see, for example, work by Bradshaw and Andrews ${ }^{45}$-leading to the following result for nonpolar molecules:

$$
\begin{aligned}
\Delta E_{\mathrm{ind}}= & \left(\frac{n \hbar c p}{\varepsilon_{0} V}\right) \operatorname{Re}\left[e_{i}^{(l)}(\boldsymbol{p}) \alpha_{i j}^{(A)}(p) V_{j k}(p, \boldsymbol{R}) \alpha_{k l}^{(B)}(p) e_{l}^{(l)}(\boldsymbol{p})\right. \\
& \times \exp (-i \boldsymbol{p} \cdot \boldsymbol{R})]
\end{aligned}
$$

Here, $n$ defines the number of real input photons, with individual energies $\hbar c p$ ( $p=2 \pi /$ laser wavelength) within the quantization volume $V$. The retarded dipole-dipole coupling tensor $V_{j k}(p, R)$ takes the same form as Eq. (13). The dynamic polarizability tensors which feature in Eq. (17) are specific implementations of the formula

$$
\alpha_{i j}^{\eta \eta(\xi)}(p)=\sum_{r}\left(\frac{\mu_{i}^{\eta r(\xi)} \mu_{j}^{r \eta(\xi)}}{E_{r \eta}^{\xi}-\hbar c p}+\frac{\mu_{j}^{\eta r(\xi)} \mu_{i}^{r \eta(\xi)}}{E_{r \eta}^{\xi}+\hbar c p}\right) \text {, }
$$

where $\eta$ designates the electronic state in which the molecule $\xi$ resides. In this expression, given here in general form with a view to the calculations in Sec. IV, omission of the state labels as in Eq. (17) signifies ground-state evaluation.

In order to fully describe the effect of optical forces on a system, it is necessary to consider internal degrees of freedom as defined by molecular geometry. Based on Eq. (17), calculations have, for example, been performed for a range of cylindrical configurations including cases of tumbling, collinear, and parallel pairs. ${ }^{44,45}$ In the case of isotropic molecules, the energy shift emerges as

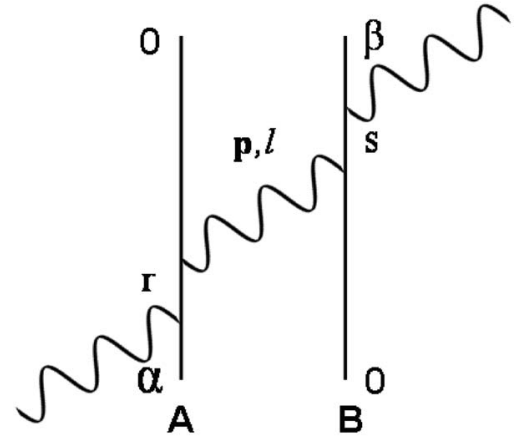

FIG. 5. One of the Feynman diagrams representing fourth-order LARET; a process mediated by a single virtual photon in the presence of throughput, off-resonant radiation assisting energy exchange between donor and acceptor.

$$
\Delta E_{\text {ind }}=\left(\frac{2 I}{\varepsilon_{0} c}\right) \operatorname{Re}\left[\alpha_{0}^{(A)} V_{x x}(p, \boldsymbol{R}) \alpha_{0}^{(B)}\right],
$$

where $x$ denotes the axis of laser polarization. The above result highlights the linear dependence on laser intensity $I$. The near-field distance dependence is accommodated within the near-field tensor element $V_{x x}(p, \boldsymbol{R})$ :

$$
\operatorname{Re}\left[V_{x x}(p, \boldsymbol{R})\right]=-\frac{1}{2 \pi \varepsilon_{0} R^{3}} .
$$

\section{Laser-assisted resonance energy transfer}

In the same way that intermolecular dispersion forces are modified by off-resonant laser light, fourth-order modifications have also been reported in connection with RET-the corresponding capacity for enhancing the rate of transfer earning the soubriquet "laser-assisted resonance energy transfer" (LARET). ${ }^{46-48}$ As with the optically induced pair forces, the throughput radiation once again emerges in a final state that is unchanged from its initial state, while in this case the material system experiences a transfer of energy from $A$ to $B$. Thus, for the initial and final states of the system as a whole we have

$$
|I\rangle=\left|A_{\alpha}, B_{0} ; n(\boldsymbol{p}, l)\right\rangle, \quad|F\rangle=\left|A_{0}, B_{\beta} ; n(\boldsymbol{p}, l)\right\rangle .
$$

It should be emphasized that the laser beam experiences no absorptive energy loss - the LARET process is not to be confused with "laser-induced RET," wherein laser frequencies are specifically chosen to promote energy migration by bridging a donor and acceptor frequency mismatch. ${ }^{49}$

Depending on how the throughput radiation interacts with the donor-acceptor system, a number of possible LARET mechanisms emerge. Each entails real photon absorption and emission, coupled by a virtual photon mediator. First, consider processes where a real photon is absorbed at the donor and subsequently re-emitted from the acceptor, see Fig. 5. The net matrix element, accommodating all time orderings, takes the following form: 


$$
\begin{aligned}
M_{F I}^{\mathrm{LARET}(1)}= & -\frac{n \hbar c p}{2 \varepsilon_{o} V}\left[e_{i}^{(l)}(\boldsymbol{p}) \alpha_{i j}^{0 \alpha(A)}(p) V_{j k}(p, \boldsymbol{R}) \alpha_{k l}^{\beta 0(B)}(p) \bar{e}_{l}^{(l)}(\boldsymbol{p})\right. \\
& \times \exp (-i \boldsymbol{p} \cdot \boldsymbol{R})+\bar{e}_{i}^{(l)}(\boldsymbol{p}) \alpha_{i j}^{0 \alpha(A)}(p) \\
& \left.\times V_{j k}(p, \boldsymbol{R}) \alpha_{k l}^{\beta 0(B)} e_{l}^{(l)}(\boldsymbol{p}) \exp (i \boldsymbol{p} \cdot \boldsymbol{R})\right] .
\end{aligned}
$$

Each term in expression (22) is mathematically isomorphous with the optically induced pair potential [Eq. (17)]—but here the process of energy transfer from $A$ to $B$ effects a differentiation between those molecules. The two parts of Eq. (22) thus reflect "mirrored" contributions-the first corresponding to the case where real photon absorption occurs at $A$ with emission at $B$, and the second, the reverse. The full LARET matrix element is completed by the inclusion of two further contributions associated with intermolecular interactions where the real photon absorption and emission processes both occur at the same center:

$$
\begin{aligned}
M_{F I}^{\mathrm{LARET}(2)}= & \frac{n \hbar c p}{2 \varepsilon_{o} V}\left[\bar{e}_{i}^{(l)}(\boldsymbol{p}) e_{l}^{(l)}(\boldsymbol{p}) \beta_{i j l}^{0 \alpha(A)}(p) V_{j k}(p ; \boldsymbol{R}) \mu_{k}^{\beta 0(B)}\right. \\
& \left.+\bar{e}_{i}^{(l)}(\boldsymbol{p}) e_{l}^{(l)}(\boldsymbol{p}) \beta_{i j l}^{\beta 0(B)}(p) V_{j k}(p, \boldsymbol{R}) \mu_{k}^{0 \alpha(A)}\right] .
\end{aligned}
$$

The hyperpolarizability tensor components $\beta_{i j l}^{f i(\xi)}$ signify the effects of three photon interactions (two real and one virtual) at a single center, being defined in the form

$$
\begin{aligned}
\beta_{j k l}^{f i(\xi)}\left(p_{3}, p_{2}, p_{1}\right)= & \sum_{r, s}\left\{\frac{\mu_{j}^{f r(\xi)} \mu_{k}^{r s(\xi)} \mu_{l}^{s i(\xi)}}{\left(E_{r i}^{(\xi)}-\hbar c p_{2}-\hbar c p_{1}\right)\left(E_{s i}^{(\xi)}-\hbar c p_{1}\right)}+\frac{\mu_{j}^{f r(\xi)} \mu_{l}^{r s(\xi)} \mu_{k}^{s i(\xi)}}{\left(E_{r i}^{(\xi)}-\hbar c p_{2}-\hbar c p_{1}\right)\left(E_{s i}^{(\xi)}-\hbar c p_{2}\right)}\right. \\
& +\frac{\mu_{k}^{f r(\xi)} \mu_{j}^{r s(\xi)} \mu_{l}^{s i(\xi)}}{\left(E_{r i}^{(\xi)}-\hbar c p_{3}-\hbar c p_{1}\right)\left(E_{s i}^{(\xi)}-\hbar c p_{1}\right)}+\frac{\mu_{k}^{f r(\xi)} \mu_{l}^{r s(\xi)} \mu_{j}^{s i(\xi)}}{\left(E_{r i}^{(\xi)}-\hbar c p_{3}-\hbar c p_{1}\right)\left(E_{s i}^{(\xi)}-\hbar c p_{3}\right)} \\
& \left.+\frac{\mu_{l}^{f r(\xi)} \mu_{j}^{r s(\xi)} \mu_{k}^{s i(\xi)}}{\left(E_{r i}^{(\xi)}-\hbar c p_{3}-\hbar c p_{2}\right)\left(E_{s i}^{(\xi)}-\hbar c p_{2}\right)}+\frac{\mu_{l}^{f r(\xi)} \mu_{k}^{r r(\xi)} \mu_{j}^{s i(\xi)}}{\left(E_{r i}^{(\xi)}-\hbar c p_{3}-\hbar c p_{2}\right)\left(E_{s i}^{(\xi)}-\hbar c p_{3}\right)}\right\} .
\end{aligned}
$$

It is interesting to observe that the above mechanism, involving the occurrence of both real photon operations at a single molecular center, also has counterparts for the optically induced pair forces. However, in the latter case the dipole moments corresponding to those in Eq. (23) are static, which in the context of the present work (addressing isotropic molecules) are zero. ${ }^{45,50}$ In LARET, the moments are associated with transition dipoles, and such terms therefore persist even for nonpolar molecules.

\section{DYNAMIC BEHAVIOR}

Intermolecular interactions are most widely understood in connection with systems in which molecules reside in their electronic ground states-a reasonable assumption when the system is in ambient conditions and electronically excited state populations are vanishingly small. Here, however, we focus on effects that are uniquely exhibited by systems in which additional electronic energy is present as a result of photoexcitation, for example. The nature of interactions between electrically neutral molecules certainly varies according to their electronic state, and those interactions are clearly subject to change during the course of absorption and RET. ${ }^{18,35}$ This is the basis for the analysis that follows.

\section{A. Effect of electronic excitation and energy transfer}

While the dispersion potential for a ground-state donoracceptor interaction is defined by Eq. (15), an ensemble of pairs incorporating donors and acceptors of any electronic $\underline{\Delta t a t e}$ generates an effective (system average) pair potential $\overline{\Delta E}$ :

$$
\overline{\Delta E}=-\frac{1}{24 \pi^{2} \varepsilon_{0}^{2} R^{6}} \sum_{\substack{a=0, \alpha, b=0, \beta}} N_{a} N_{b} \sum_{\substack{r=0, \alpha, \alpha^{*}, s=0, \beta, \beta^{*}}} \frac{\left|\boldsymbol{\mu}^{a r(A)}\right|^{2}\left|\boldsymbol{\mu}^{b s(B)}\right|^{2}}{E_{r a}^{A}+E_{s b}^{B}} .
$$

In the first summation on the right-hand side of Eq. (25), $N_{a}$ and $N_{b}$ are the fractional populations of donors in state $a$ and acceptors in state $b$, respectively, whose explicit time dependences produce dynamical effects on $\overline{\Delta E}$-as will emerge from subsequent population modeling in Sec. IV B. The second summation in Eq. (25) is taken over donor and acceptor molecular states, each molecule being treated as a three-level system to reflect its most prominent optical features. For the generic state labels we have $r \in\left\{0, \alpha, \alpha^{*}\right\}$ and $s \in\left\{0, \beta, \beta^{*}\right\}$, perturbation theory precluding the combination $a=r$ and $b=s$. The higher energy states $A_{\alpha^{*}}$ and $B_{\beta^{*}}$ are included as representatives of unpopulated (virtual) electronic states. The physical significance of the different forms that arise for the summand interactions in Eq. (25) is that the energy denominator can, according to the pair states for which it is evaluated, yield a negative result. Bearing in mind the sign at the front of Eq. (25) and the overall dependence on an inverse power of $R$, it transpires that the potential in such cases no longer describes an attractive force, as by contrast is always the case for neutral molecules. ${ }^{40}$

\section{B. Time-dependent system}

A sequence of photophysical interactions engaging the ensemble pairs is used to evaluate the time-evolving populations $N_{a}$ and $N_{b}$, subsequently to be used in determining temporal changes in the ensemble dispersion as a result of 
energy transfer. The initial state preparation is effected by an initial excitation of donor molecules through the absorption of light that is resonant with the donor but not the acceptor. The donor excitation leads to a population of excited vibrational levels (denoted by double dagger superscript) of the electronic excited state $A_{\alpha}$. While laser excitation might result in localized movement as a result of radiation pressure, such movement can be ignored in the following calculations; beyond initial excitation, the input plays no further part in subsequent events. Without compromising energy conservation by the system as a whole, an immediate consequence of electronic excitation is the partial dissipation of electronic energy through coupling to nuclear vibrations-the usual process of intramolecular vibrational relaxation (IVR), assigned the rate constant $k_{\mathrm{IVR}}$. In the analysis that follows, it is assumed that IVR reaches effective completion prior to subsequent relaxation to the electronic ground state, the latter proceeding through a variety of mechanisms including spontaneous emission. For simplicity, all such electronic relaxation processes of the donor, with the exception of RET, are included in a representative rate constant $k_{\text {rel }}^{\alpha}$. The separate distinction of RET is necessary because energy migration populates the vibrational levels of $B_{\beta}$.

Prior to energy transfer, the preceding IVR will generally place the donor molecule in an energy level where its decay profile has a relatively small overlap with the red end of any neighboring donor's absorption. In the process of RET to a nearby acceptor, however, a much larger spectral overlap and hence a significantly larger transfer rate will generally apply, so that energy transfer to acceptors will be the dominant process. Moreover, the spectroscopic gradient $\left(E_{\alpha 0}^{A}>E_{\beta 0}^{B}\right)$ commonly associated with donor-acceptor transfer will engender a high degree of directed character, such that "backward" transfer of energy (acceptor to donor) can be ignored. ${ }^{51}$ The final process undergone by the system is vibrational and electronic relaxation of the acceptor. Here, all relaxation processes are accommodated by the representative rate constant $k_{\text {rel. }}^{\beta}$.

Time-resolved changes in the population of the initial excited state can be determined by analysis of all decay routes of $A_{\alpha}$, as shown in Fig. 6 ,

$$
\frac{d}{d t} N_{\alpha}=-\left(k_{\mathrm{rel}}^{\alpha}+k_{\mathrm{RET}}\right) N_{\alpha} .
$$

The above differential equation is solved with the initial population of $A_{\alpha}, N_{\alpha}(0)$, assigned an arbitrary value, such that

$$
N_{\alpha}(t)=N_{\alpha}(0) \exp \left(-t\left(k_{\mathrm{rel}}^{\alpha}+k_{\mathrm{RET}}\right)\right) .
$$

Applying the physically reasonable conditions $k_{\mathrm{IVR}}^{\beta^{\ddagger}}>k_{\mathrm{RET}}$, $N_{\alpha}>N_{\beta^{\ddagger}}^{\ddagger}$, and $N_{\beta}(0)=0$, the growth of $N_{\beta}$ is dependent solely on RET from the ground vibrational state of $A_{\alpha}$. Furthermore, the time-dependent variation in $N_{\beta}$ is represented by the following expression:

$$
\frac{d}{d t} N_{\beta}=k_{\mathrm{RET}} N_{\alpha}-k_{\mathrm{rel}}^{\beta} N_{\beta} .
$$

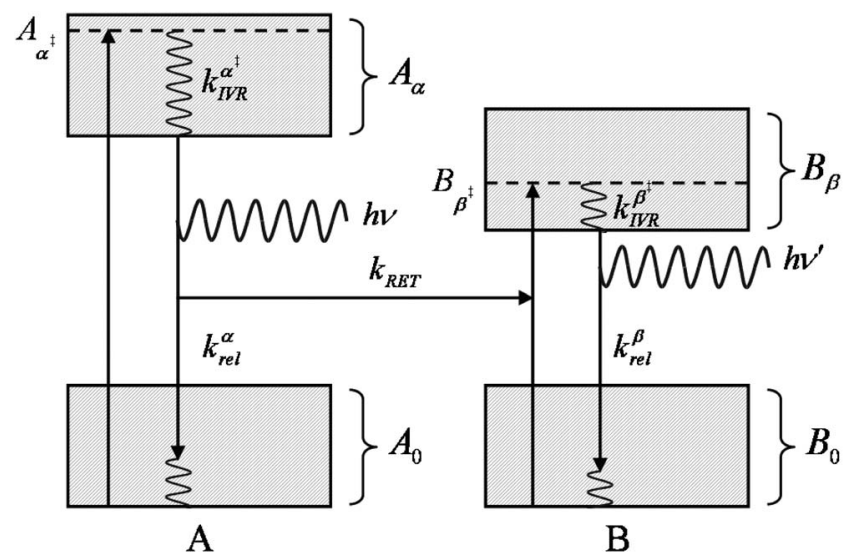

FIG. 6. Jablonski diagram, rate constants $k$ denoting processes determining the excited populations of molecules $A$ and $B$. These processes involve among others, the spontaneous emission of photons $h \nu$ from $A_{\alpha}$ and lower energy emission (owing to IVR) of $h \nu^{\prime}$ from $B_{\beta}$.

In the presence of an auxiliary off-resonant beam, the LARET effect will significantly enhance the rate of donoracceptor energy transfer compared to second-order RET. To represent the rate increase due to LARET an additional rate term, cast in terms of a constant $k_{\mathrm{LARET}}$, is introduced to the kinetics of the donor molecule. In dealing with a laser pulse of sufficiently short duration, i.e., comparable to the modeled excited state lifetimes, the time-dependent behavior of the off-resonant pulse must also be considered. We choose to modulate the rate constant $k_{\text {LARET }}$ by adopting a pulse shape of the form $f(t)=\operatorname{sech}^{2}\left[\omega\left(t-t^{\prime}\right)\right]$, with $\omega$ being proportional to the pulse width. The donor excited state decay, accounting for the effects of both RET and LARET, is now represented by

$$
\frac{d}{d t} N_{\alpha}=-\left(k_{\mathrm{rel}}^{\alpha}+k_{\mathrm{RET}}+\operatorname{sech}^{2}\left[\omega\left(t-t^{\prime}\right)\right] k_{\mathrm{LARET}}\right) N_{\alpha} .
$$

The corresponding rate of acceptor excitation is as follows:

$$
\frac{d}{d t} N_{\beta}=\left(k_{\mathrm{RET}}+\operatorname{sech}^{2}\left[\omega\left(t-t^{\prime}\right)\right] k_{\mathrm{LARET}}\right) N_{\alpha}-k_{\mathrm{rel}}^{\beta} N_{\beta} .
$$

Finally, the optically induced pair forces must also be considered dependent on time with respect to the operation of the laser pulse. By substitution of Eqs. (18) and (20) into Eq. (19) the temporal behavior of the corresponding optically induced pair potential is

$$
\begin{aligned}
\Delta E_{\text {ind }}= & -\sum_{r, s}\left(\left(\frac{\operatorname{sech}^{2}\left[\omega\left(t-t^{\prime}\right)\right] I}{9 \pi \varepsilon_{0}^{2} c R^{3}}\right)\left(\frac{\left|\boldsymbol{\mu}_{A}^{a r}\right|^{2}}{E_{r a}^{A}-\hbar c p}+\frac{\left|\boldsymbol{\mu}_{A}^{a r}\right|^{2}}{E_{r a}^{A}+\hbar c p}\right)\right. \\
& \left.\times\left(\frac{\left|\boldsymbol{\mu}_{B}^{b s}\right|^{2}}{E_{s b}^{B}-\hbar c p}+\frac{\left|\boldsymbol{\mu}_{B}^{b s}\right|^{2}}{E_{s b}^{B}+\hbar c p}\right)\right),
\end{aligned}
$$

the factor of 9 in the denominator arising as a result of isotropic averaging.

\section{RESULTS}

To quantify the dynamically evolving energy flow within an ensemble, the fractional populations $N_{\alpha}$ and $N_{\beta}$ are first determined from Eqs. (29) and (30), the results being shown 

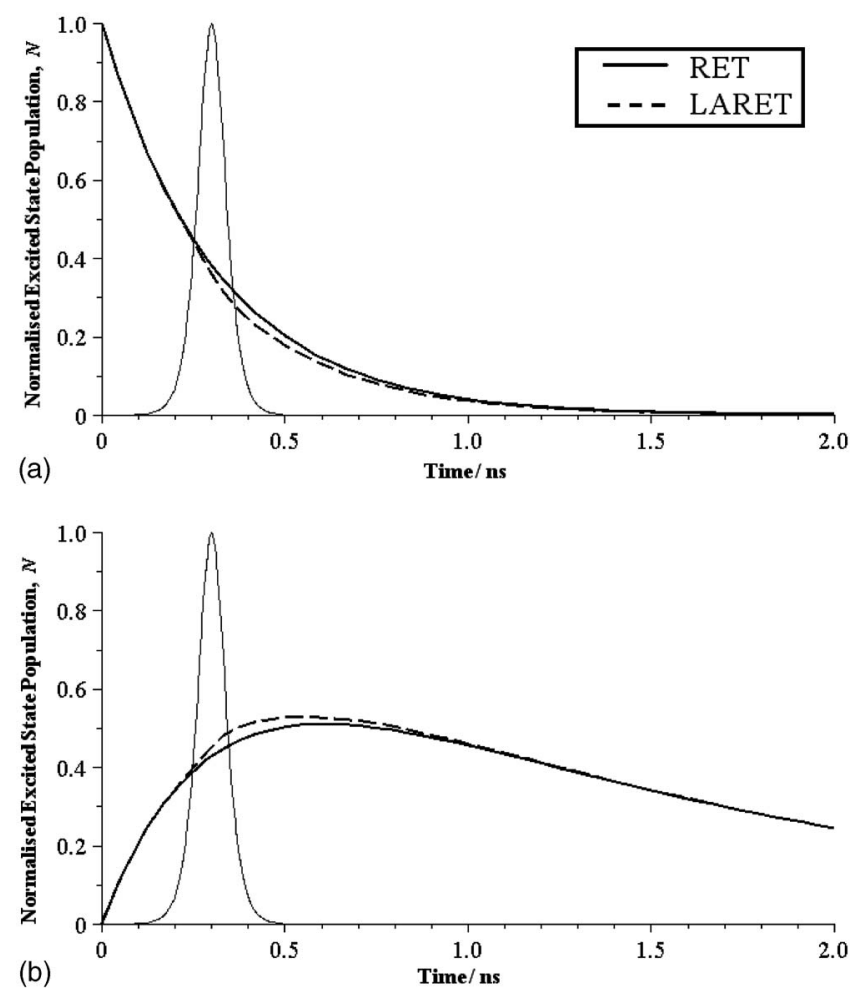

FIG. 7. Representative $N_{\alpha}$ and $N_{\beta}$ population profiles, (a) and (b), respectively, following initial pulsed laser excitation. Values of $k_{\mathrm{RET}}$ and $k_{\text {rel }}^{\alpha}$ are chosen such that $k_{\mathrm{RET}}$ accounts for approximately $80 \%$ of the total decay of $N_{\alpha}$. Total rate of energy transfer is modestly increased by $50 \%$ through $k_{\mathrm{LARET}} ; k_{\mathrm{rel}}^{\alpha}$ and $k_{\text {rel }}^{\beta}$ are taken as equal.

in Fig. 7. It is assumed that there is a sufficiently strong short-range interaction between each donor and acceptor that the electronic state decay kinetics will be primarily determined by fast energy transfer; the donor lifetime is arbitrarily set as 2 ns. Since it is only necessary to consider relative populations when addressing the temporal form of the intermolecular potentials, an arbitrary initial value of unity can be assigned to $N_{\alpha}(0)$ - this does not signify an initial fully populated electronic excited state.

In the subsequent determination of numerical results for the system energy, from Eqs. (25) and (31), the transition dipole moments $\boldsymbol{\mu}^{a r(A)}$ and $\boldsymbol{\mu}^{b s(B)}$ were both set as $5 \mathrm{D}$ and the donor-acceptor intermolecular distance as $1.0 \mathrm{~nm}$. The transition energies to $A_{\alpha^{*}}$ and $A_{\alpha}$ from $A_{0}$ were chosen to correspond with wavelengths of 300 and $350 \mathrm{~nm}$, respectively. Lower energies were chosen for the transitions from $B_{0}$ to $B_{\beta^{*}}$ and $B_{\beta}$, associated with wavelengths of 400 and $450 \mathrm{~nm}$ accordingly. The LARET input, off-resonant at a wavelength of $500 \mathrm{~nm}$, was modeled as a laser pulse having a duration of $100 \mathrm{ps}$ (full width at half maximum), being delivered to the system with a delay of approximately $0.3 \mathrm{~ns}$ after the initial donor excitation-see Fig. 7.

The ensemble-averaged variation in the donor-acceptor pair interaction energy is exhibited in Fig. 8. Following the donor electronic excitation, a decrease in $N_{\alpha}$ through relaxation and energy transfer establishes a corresponding decrease in the pair energy. It is predicted in this example that the interaction energy reaches a minimum as $N_{\beta}$ approaches a maximum, after which the system reverts to its ground-

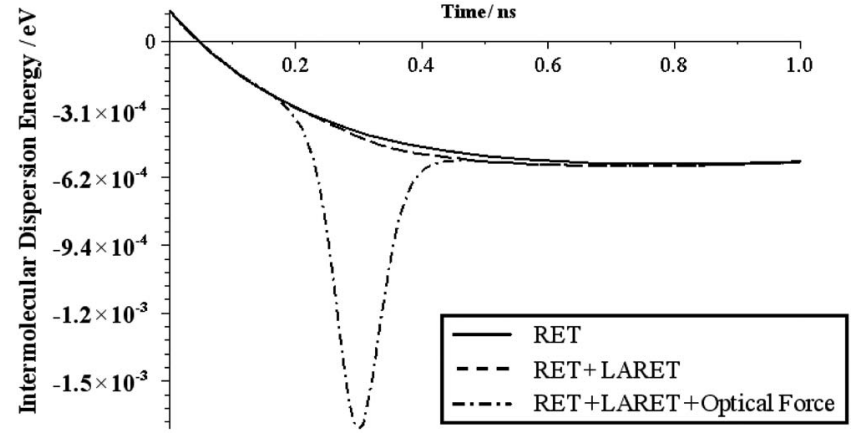

FIG. 8. Evolution of the optically modified donor-acceptor interaction as a function of time, highlighting system response to RET, LARET, and optically induced forces.

state interaction potential. ${ }^{18}$ A striking feature of the result, comparing the relative significance of fourth-order interactions, is that optically induced forces are very much more significant in their effect than LARET.

\section{PHYSICAL MANIFESTATIONS}

In the wide context of experimental studies based on FRET, many applications concern the determination of molecular conformational change-using fluorescence lifetime measurements with suitable tags for the study of single molecule protein folding, for example. ${ }^{52-54}$ It is rarely considered that the transfer of energy itself might stimulate or modify such changes. Any variation in intermolecular pair potential, as determined in the present analysis, must invariably result in a localized movement that, to a degree, either closes or expands the distance between any donor and its counterpart acceptor. While it is relatively simple to develop from the change in interaction energy a corresponding ensembleaveraged force, the values typically varying in the piconewton range ${ }^{18}$ such results can only be regarded as indicative. The theoretical evaluation of a measurable displacement remains complex, but it is in principle achievable for any chemical system of interest, through the deployment of a suitable molecular modeling package.

One other area in which the effects we describe may prove of particular significance is in the development of micro- and nanoelectromechanical systems. These devices represent a rapidly developing technology that is already being used in sensors and actuators for a variety of applications, including integrated drug delivery systems $\mathrm{s}^{55,56}$ and optical scanners. ${ }^{57,58}$ In such connections there is considerable interest in harnessing the variations in interatomic and intermolecular displacements that can arise as a result of quantum (Casimir force) effects-forces that become especially prominent as the size of such devices shrinks down to nanoscale dimensions. ${ }^{59}$ Our work shows that the engagement of energy transfer between the components in such devices can offer additional means for effecting mechanical motion. There is a distinct possibility of introducing optical force control over nanoscale motions. 


\section{ACKNOWLEDGMENTS}

The authors are indebted to EPSRC for the DTA financial support. We also thank Dr. Luciana Dávila Romero for helpful comments.

\section{APPENDIX: DISPERSION POTENTIAL FROM DIPOLE COUPLING}

Traditionally, intermolecular dispersion energies as presented in Eq. (15) are derived using second-order perturbation theory and the well-known dipole-dipole pair interaction operator, $\mathbf{W}_{A B}$, which has the form

$$
\mathbf{W}_{A B}=\frac{\mu_{i}^{(A)} \mu_{j}^{(B)}}{4 \pi \varepsilon_{0} R^{3}}\left(\delta_{i j}-3 \hat{R}_{i} \hat{R}_{j}\right) .
$$

Here, $\mathbf{W}_{A B}$ is the quantum operator corresponding to a classical energy - which, in the case of static dipoles, would be determined by substitution of Eq. (10) into Eq. (9). The result is explicitly unretarded; moreover the same form applies for any form of near-field dipolar interaction. Based on Eq. (A1), the short-range form of dispersion interaction emerges directly from second-order perturbation theory, such that

$$
\Delta E=\sum_{R} \frac{\left\langle F\left|\mathbf{W}_{A B}\right| R\right\rangle\left\langle R\left|\mathbf{W}_{A B}\right| I\right\rangle}{E_{I}-E_{R}} .
$$

On substitution of Eq. (A1) into Eq. (A2), with the state of each component duly specified, the pair dispersion is given by

$$
\Delta E\left(A_{a} B_{b}\right)=\sum_{r, s} \frac{\left\langle A_{a} ; B_{b}\left|\mathbf{W}_{A B}\right| A_{r} ; B_{s}\right\rangle\left\langle A_{r} ; B_{s}\left|\mathbf{W}_{A B}\right| A_{a} ; B_{b}\right\rangle}{E_{a}^{A}+E_{b}^{B}-E_{r}^{A}-E_{s}^{B}}=\frac{1}{16 \pi^{2} \varepsilon_{0}^{2} R^{6}}\left[\frac{\mu_{i}^{a r(A)} \mu_{j}^{b s(B)} \mu_{k}^{r a(A)} \mu_{l}^{s b(B)}\left(\delta_{i j}-3 \hat{R}_{i} \hat{R}_{j}\right)\left(\delta_{k l}-3 \hat{R}_{k} \hat{R}_{l}\right)}{E_{a r}^{A}+E_{b s}^{B}}\right] .
$$

While generally valid for rigidly oriented molecules, the key features of the physics are clarified by performing an orientational average; the dipole moments of both the donor and acceptor are treated as being randomly oriented in situ, resulting in Eq. (15) - the well-known London formula, when $a$ and $b$ are ground levels.

${ }^{1}$ Resonance Energy Transfer, edited by D. L. Andrews and A. A. Demidov (Wiley, Chichester, 1999).

${ }^{2}$ X. Hu, A. Damjanovic, T. Ritz, and K. Schulten, Proc. Natl. Acad. Sci. U.S.A. 95, 5935 (1998)

${ }^{3}$ R. J. Cogdell, A. T. Gardiner, A. W. Roszak, C. J. Law, J. Southall, and N. W. Isaacs, Photosynth. Res. 81, 207 (2004)

${ }^{4}$ V. Balzani, A. Credi, and M. Venturi, ChemSusChem 1, 26 (2008).

${ }^{5}$ M. Takahashi, H. Morimoto, Y. Suzuki, T. Odagi, M. Yamashita, and H. Kawai, Tetrahedron 60, 11771 (2004).

${ }^{6}$ A. Zabala Ruiz, H. Li, and G. Calzaferri, Angew. Chem. 118, 5408 (2006)

${ }^{7}$ D. L. Andrews, S. Li, J. Rodríguez, and J. Slota, J. Chem. Phys. 127, 134902 (2007)

${ }^{8}$ A. Hepp, G. Ulrich, R. Schmechel, H. von Seggern, and R. Ziessel, Synth. Met. 146, 11 (2004)

${ }^{9}$ S. Zhang, Z. Lu, Y. Peng, Y. Liu, and Y. Yang, J. Lumin. 128, 1523 (2008)

${ }^{10}$ S. Saini, H. Singh, and B. Bagchi, J. Chem. Sci. 118, 23 (2006).

${ }^{11}$ K. E. Sapsford, L. Berti, and I. L. Medintz, Angew. Chem. 45, 4562 (2006).

${ }^{12}$ Y. R. Khan, T. E. Dykstra, and G. D. Scholes, Chem. Phys. Lett. 461, 305 (2008).

${ }^{13}$ P. I. H. Bastiaens and A. Squire, Trends Cell Biol. 9, 48 (1999).

${ }^{14}$ G. Colas des Francs, C. Girard, and O. J. F. Martin, Phys. Rev. A 67, 053805 (2003)

${ }^{15}$ G. Colas des Francs, C. Girard, M. Juan, and A. Dereux, J. Chem. Phys. 123, 174709 (2005).

${ }^{16}$ R. R. Duncan, Biochem. Soc. Trans. 34, 679 (2006).

${ }^{17}$ E. A. Jares-Erijman and T. M. Jovin, Curr. Opin. Chem. Biol. 10, 409 (2006).
${ }^{18}$ D. L. Andrews, D. S. Bradshaw, J. M. Leeder, and J. Rodriguez, Phys. Chem. Chem. Phys. 10, 5250 (2008).

${ }^{19}$ C. Ates, A. Eisfeld, and J. M. Rost, New J. Phys. 10, 045030 (2008).

${ }^{20}$ W. P. Healy, Non-Relativistic Quantum Electrodynamics (Academic, London, 1982).

${ }^{21}$ D. P. Craig and T. Thirunamachandran, Molecular Quantum Electrodynamics (Dover, New York, 1998)

${ }^{22}$ A. Salam, Int. Rev. Phys. Chem. 27, 405 (2008).

${ }^{23}$ G. W. Drake, Nucl. Phys. A. 737, 25 (2004).

${ }^{24}$ S. G. Karshenboim, Phys. Rep. 422, 1 (2005).

${ }^{25}$ P. H. Mokler, Radiat. Phys. Chem. 75, 1730 (2006).

${ }^{26}$ G. Gabrielse, D. Hanneke, T. Kinoshita, M. Nio, and B. Odom, Phys. Rev. Lett. 97, 030802 (2006).

${ }^{27}$ C. I. Sukenik, M. G. Boshier, D. Cho, V. Sandoghdar, and E. A. Hinds, Phys. Rev. Lett. 70, 560 (1993).

${ }^{28}$ D. L. Andrews, N. P. Blake, and K. P. Hopkins, J. Chem. Phys. 88, 6022 (1988)

${ }^{29}$ D. L. Andrews and N. P. Blake, J. Mod. Opt. 37, 701 (1990).

${ }^{30}$ D. L. Andrews and P. Allcock, Chem. Phys. 198, 35 (1995).

${ }^{31}$ D. L. Andrews and P. Allcock, Optical Harmonics in Molecular Systems: Quantum Electrodynamical Theory (Wiley-VCH, Weinheim, 2002).

${ }^{32}$ G. D. Scholes and D. L. Andrews, J. Chem. Phys. 107, 5374 (1997).

${ }^{33}$ A. Salam, J. Chem. Phys. 122, 044112 (2005).

${ }^{34}$ E. A. Power and T. Thirunamachandran, Phys. Rev. A 48, 4761 (1993).

${ }^{35}$ A. E. Cohen and S. Mukamel, J. Phys. Chem. A 107, 3633 (2003).

${ }^{36}$ D. L. Andrews and D. S. Bradshaw, Eur. J. Phys. 25, 845 (2004).

${ }^{37}$ P. O. Löwdin, Perturbation Theory and its Applications in Quantum Mechanics (Wiley, New York, 1965).

${ }^{38}$ G. J. Daniels, R. D. Jenkins, D. S. Bradshaw, and D. L. Andrews, J. Chem. Phys. 119, 2264 (2003).

${ }^{39}$ G. Juzeliūnas and D. L. Andrews, in Resonance Energy Transfer, edited by D. L. Andrews and A. A. Demidov (Wiley, Chichester, 1999), p. 65.

${ }^{40}$ E. A. Power and T. Thirunamachandran, Phys. Rev. A 51, 3660 (1995).

${ }^{41}$ B. W. Alligood and A. Salam, Mol. Phys. 105, 395 (2007).

${ }^{42}$ H. B. G. Casimir and D. Polder, Phys. Rev. 73, 360 (1948).

${ }^{43}$ D. L. Andrews, R. G. Crisp, and D. S. Bradshaw, J. Phys. B 39, S637 (2006).

${ }^{44}$ L. C. Dávila Romero and D. L. Andrews, in Structured Light and Its Applications: An Introduction to Phase-Structured Beams and Nanoscale 
Optical Forces, edited by D. L. Andrews (Elsevier, Amsterdam, 2008), p. 79.

${ }^{45}$ D. S. Bradshaw and D. L. Andrews, Phys. Rev. A 72, 033816 (2005).

${ }^{46}$ P. Allcock, R. D. Jenkins, and D. L. Andrews, Chem. Phys. Lett. 301, 228 (1999).

${ }^{47}$ P. Allcock, R. D. Jenkins, and D. L. Andrews, Phys. Rev. A 61, 023812 (2000).

${ }^{48}$ D. L. Andrews, Can. J. Chem. 86, 855 (2008).

${ }^{49}$ A. Bambini, Phys. Rev. A 53, 3302 (1996).

${ }^{50}$ A. Salam, Phys. Rev. A 76, 063402 (2007).

${ }^{51}$ D. L. Andrews and J. Rodríguez, J. Chem. Phys. 127, 084509 (2007).

${ }^{52}$ S. Weiss, Nat. Struct. Biol. 7, 724 (2000).
${ }^{53}$ B. Schuler, E. A. Lipman, and W. A. Eaton, Nature (London) 419, 743 (2002).

${ }^{54}$ A. Borgia, P. M. Williams, and J. Clarke, Annu. Rev. Biochem. 77, 101 (2008).

${ }^{55}$ N. C. Tsai and C. Y. Sue, Sens. Actuators, A 134, 555 (2007).

${ }^{56}$ A. Nisar, N. Afzulpurkar, B. Mahaisavariya, and A. Tuantranont, Sens. Actuators B 130, 917 (2008).

${ }^{57}$ S. Kim, G. Barbastathis, and H. L. Tuller, J. Electroceram. 12, 133 (2004).

${ }^{58}$ J. M. Zara and S. W. Smith, Sens. Actuators, A 102, 176 (2002).

${ }^{59}$ H. B. Chan, V. A. Aksyuk, R. N. Kleiman, D. J. Bishop, and F. Capasso, Science 291, 1941 (2001). 